\title{
Major Energy Efficient Routing Schemes in Wireless Sensor Networks: Survey and Idea
}

Jahangeer Ali, Gulshan Kumar, Dr. Mritunjay Kumar Rai

Department of CSE, Lovely Professional University, Punjab Jaims7575@gmail.com

Department of CSE, Lovely Professional University, Punjab

gulshan.16865@Ipu.co.in

Department of CSE, Lovely Professional University, Punjab

raimritunjay@gmail.com

\section{ABSTRACT}

Sensing the environment without human intervention is carried out with Wireless Sensor Networks. Thus WSNs have gained impetus in every field as applicable to various sensing applications. As the sensor nodes are very minute with limited power, memory and controlling mechanism. Thus it is necessary to implement energy efficient routing in sensor nodes such that network lifetime is enhanced. In this paper, we have discussed various existing energy efficient routing schemes and made comparison on various parameters in literature survey. Finally came to conclusion that there is a need of an energy efficient routing protocol which can further extend network lifetime. We propose an idea in which existing; Enhanced Energy Efficient Protocol with Static Clustering (EEEPSC) is modified by placing a fraction of nodes having more energy than normal nodes in the locations where Base Station is far away. And BS is placed within the area of deployed nodes.

\section{Indexing terms}

Wireless sensor network, Network lifetime, Clustering, Cluster head (CH).

\section{INTRODUCTION}

Wireless Sensor Network (WSN) has came forth as an important new field in wireless communication. Due to recent advances in Micro-Electronic- Mechanical Systems (MEMS) and wireless communication technologies, sensors are deployed in a physical environment and communicating through wireless links and thus provide new opportunities for a variety of civilian and military applications, for example, environmental monitoring, battle field surveillance, and industry process control [1]. The basic unit of WSN is sensor node. Sensor Nodes monitor physical parameters such as temperature, pressure, motion or vibration, pollutants etc. The sensor node performs three primary functions sensing, communicating and data processing [2]. The biggest challenge in WSN is the limited battery energy as these are deployed in areas where it is not feasible to replace the battery. Thus energy utilization and preservation is prime concern of most of the researchers. Each sensor node requires battery backup because it consists of CPU, sensing unit and transmitting unit (radio) which require energy in order to carry out operations. Energy management in WSNs not only preserves the energy of sensor nodes but increasing the longevity of the whole network. The wireless sensor networks consist of thousands of sensor nodes that are densely deployed in the target environment to collect the messages and transmitting information to the sink (Base Station). Internet addressing will not be applicable to WSNs because sensor nodes are in huge in number and it is not possible to maintain or store the IP addresses in their corresponding tables. Thus routing process is data centric in WSN. Routing algorithm in the WSN has been classified into three main categories [3]:

(1) Flat routing

(2) Hierarchical routing

(3) Location based routing

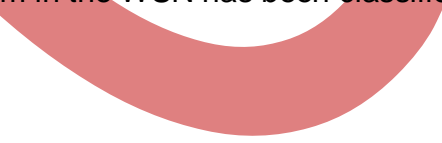

In flat routing all nodes have same functionalities and performing same task. Data transmission is hop by hop like flooding and direct diffusion. The main drawbacks of flat routing are limited to small networks only, no aggregation. In location based routing, sensor nodes are aware about their locations enabled by GPS based system.

Hierarchical routing based schemes are more scalable and efficient in communication. Clusters are formed and in each cluster a Cluster Head $(\mathrm{CH})$ is chosen with high residual energy. The nodes directly transfer data to $\mathrm{CH}$ with aggregates data and transmits it to sink node. They consume less energy because of data aggregation which helps in reducing the number of transmitted messages to base station. Hierarchical routing has somehow managed to be most energy efficient routing protocols for WSNs [4]. Most of the researchers have worked on clustering protocols to save the limited energy in order to increase the network life. Clustering algorithms like LEACH [5], LEACH-C [7], PEGASIS [8], and HEED [10] have improved the energy efficiency of WSNs. However the energy utilized in dynamic cluster formation will lead to more overhead which will directly affect the network lifetime of WSN. It is very important to maintain equilibrium between data delivery, network lifetime, data aggregation in order to come out with the energy efficient scheme in WSNs.

In this paper, we will put forth various clustering based routing techniques which are explained in literature survey. We will concentrate on most efficient published schemes and list some shortcomings exist in these protocols. And finally a comparison table is given which based on various parameters easily explains them. 


\section{LITERATURE SURVEY}

W.R. Heinzelman, A.P. Chandrakasan and H.Balakrishnan [5] proposed Low Energy Adaptive Clustering Hierarchy $(\mathrm{LEACH})$ protocol. It is first energy efficient routing protocol for hierarchical clustering. It increases the network life by reducing the overall energy consumption of WSNs. In LEACH Protocol clusters are formed and for each cluster, a Cluster head is selected in a random way. The non cluster head nodes sense the data and forward it to cluster heads. The cluster heads aggregate the received data and the forward the data to the sink. This aggregation process reduces the transmission of redundant data. Cluster heads change randomly over time in order to balance the energy dissipation of nodes. This decision is made by the node choosing a random number between 0 and 1 . The node becomes a cluster head for the current round if the number is less than the following threshold:

$$
T(n)=\left\{\begin{array}{cc}
\frac{p}{1-p(r \bmod 1 / p)} & \text { if } n \in G \\
0 & \text { otherwise }
\end{array}\right.
$$

Where $P=$ the desired percentage of cluster heads (e.g. $P=0.05), r=$ current round $G=$ set of nodes that have not been the cluster heads in last1/P rounds.

There are two phases in LEACH protocol: i) Setup phase ii) Steady-state phase. In the setup phase the clusters are formed and the cluster-heads are selected. In the steady-state phase, the data from non cluster heads are transmitted to the sink. The sensor nodes communicate to the cluster-heads using TDMA schedule. Sensor nodes forward data to $\mathrm{CHs}$ in their allotted time slots. Thus avoids collision.

There should be no confusion about the existence of $\mathrm{LEACH}$ in these surveys. As most of existing energy efficient protocols are based on LEACH. Due to advances in routing, LEACH protocol has some limitation that it uses single-hop routing in intra and inter-clustering communication. Thus not applicable to networks deployed in large regions, and the cluster head selected randomly and thus difficult to optimize.

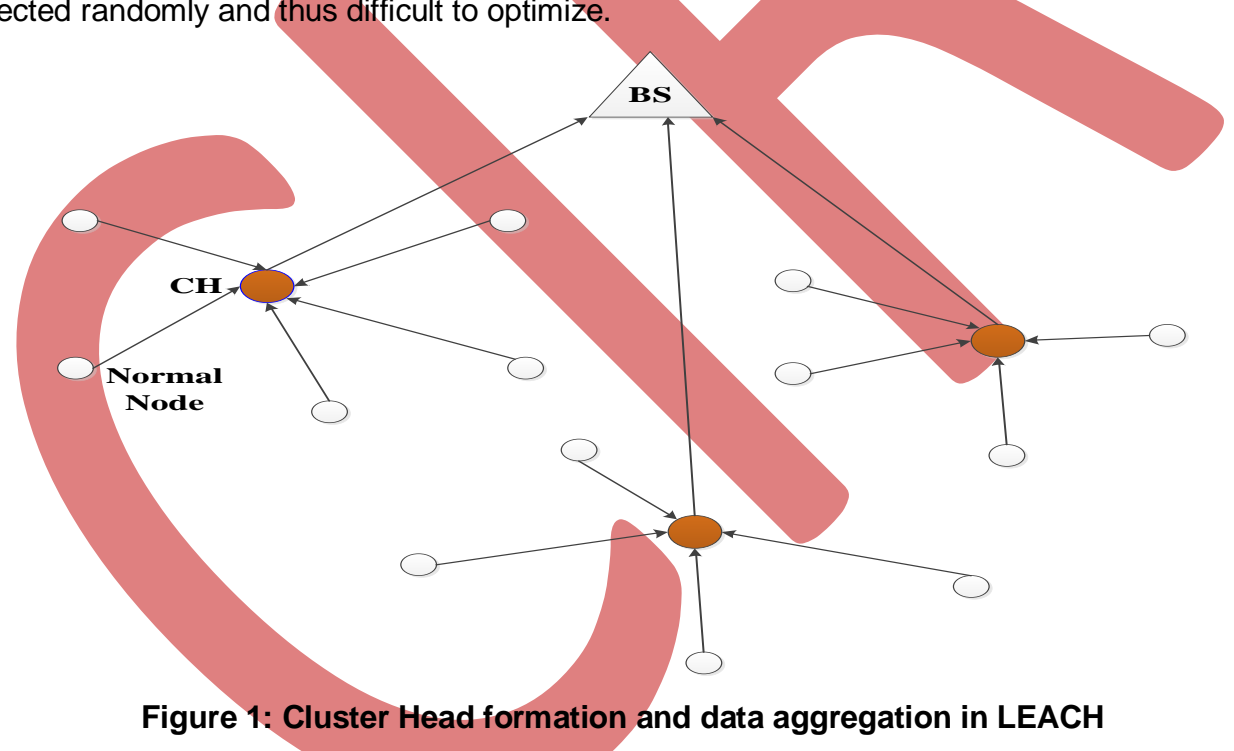

In 2002, M. Younis, M. Youssef and K. Arisha [6] proposed An Energy Aware Routing scheme called EAR. In Energyaware routing nodes are grouped into clusters. Cluster heads namely gateways are less energy constrained nodes. Gateways maintain the states of the nodes and sets up multi hop routes. Sink only communicates with the gateway. Gateway informs other nodes about in which slot they should listen, others transmission in which slot they can use for transmission. A cost function is defined between any two nodes in terms of energy consumption, delay optimization and other performance metrics. Using this cost function, a least-cost path is found between sensor nodes and the gateway.

In 2011, M.F.K. Abad, M.A.J.Jamali [7] proposed modify LEACH Algorithm for Wireless Sensor Networks (LEACH-C) uses a centralized clustering algorithm and same steady-state protocol. During the set-up phase of LEACH-C, each node sends information about current location and energy level to base station (BS). The BS will determine clusters, $\mathrm{CH}$ and non-CHs of each cluster. The BS utilizes its global information of the network to produce better clusters that require less energy for data transmission. The number of $\mathrm{CH}$ s in each round of $\mathrm{LEACH}-\mathrm{C}$ equals a predetermined optimal value.

In 2002, S. Lindsey and C. Raghavendra [8] proposed Power Efficient Gathering in Sensor Information Systems (PEGASIS) protocol. It is an enhanced version of LEACH protocol. That is a chain based protocol provides improvement over LEACH algorithms. Each node aggregates the collected data with its own data and then passes the aggregated data to the next node in the chain and finally to the designate node which transmits it to BS. Using greedy algorithm, the nodes will be organized to form a chain, after that BS can compute this chain and broadcast it to all the sensor nodes. Energy saving in PEGASIS over LEACH takes place by many stages [4]: First, in the local data gathering, the distances that most of the sensor nodes transmit are much less compared to transmitting to a cluster-head in LEACH. Second, only one node transmits to the BS in each round of communication. Also the number of transmissions to BS is 
reduced. It employs multi hop transmission and selecting only one node to transmit the data to the sink or base station while in LEACH it uses single hop. Since the overhead caused by dynamic cluster formation is eliminated.

For larger networks, PEGASIS adds excessive delay for distant nodes. And the single designated node can be a bottleneck which is responsible to transmit the data to BS. It is based on some assumptions which make solutions not practical in real world like any node can send information directly to base station. And all nodes are aware of locations of sensor nodes in WSN.

In 2011, P. Chutima, M. Sujitra proposed [09] Optimal WSN Design for Efficient Energy Utilization. In the paper, the authors necessitated the need of installing the new fewer nodes as Relay Nodes. These relay nodes may be equipped with more sophisticated energy sources such as solar cells with larger batteries. The main aim was to solve the relay station placement and assignment problem (RPAP) for WSN.The sensor nodes will transmit the sensing information to the suitable RS. The proposed mode aims to determine routes for transmitting this information so that the resulting network can guarantee the required network lifetime and ensure the radio communication between sensor nodes so that network can guarantee packet delivery from SNs to base station.

In 2004, O. Younis and S. Fahmy proposed [10] HEED (Hybrid Energy-Efficient Distributed clustering).It is somehow based on the concept of LEACH by using the residual energy and network topology features (e.g. node degree) in cluster selection to achieve energy balancing. It is based on hybrid approach, cluster head selection is based randomly as it was in LEACH. But the nodes will join only the particular cluster whose communication cost is minimum. It uses multi hop topology, using an adaptive transmission power in the inter-clustering communication. It terminates in constant number of iterations, independent of network diameter. These features make HEED more energy efficient than existing schemes already given before.

The major limitations in HEED protocol are [4]: (i) the use of tentative $\mathrm{CHs}$ that do not become final $\mathrm{CHs}$ leave some uncovered nodes. As per HEED implementation, these nodes are forced to become a $\mathrm{CH}$ and these forced $\mathrm{CH}$ may be in range of other $\mathrm{CHs}$ or may not have any member associated with them. As a result, more $\mathrm{CHs}$ are generated than the expected number and this also accounts for unbalanced energy consumption in the network (ii) The CHs near the sink have more work load so may die early. (iii) Overhead occurs as several iterations are performed to form clusters and lots of packets are broadcasted in each iteration.

In 2005M. YE, C. LI, G. CHEN, and J. WU proposed [13] An Energy Efficient Clustering Scheme in Wireless Sensor Networks (EECS).It is clustering based LEACH protocol operates in single hop mode between $\mathrm{CH}$ and BS. Sensor nodes compete for the ability to become $\mathrm{CH}$ for a given round. It involves sensor nodes into competition by broadcasting their residual energy to neighboring candidates. If a given node does not find a node with more residual energy, it becomes a $\mathrm{CH}$. The main distinguishing feature from LEACH is the dynamic sizing used in clustering in which cluster distance from base station is computed. This scheme is mostly used in periodical data gathering applications in WSNs.

However EECS possesses some limitations like: (i) It uses single hop networks and can consume more energy for longer distances between $\mathrm{CHs}$ and BS. (ii) As all nodes compete for elevating to $\mathrm{CH}$ will add more complexity overhead.(iii) Overhead due to more global knowledge about distances between BS and CHs.

In 2001, Anjeshwar and Agrawal proposed [12] Threshold sensitive Energy Efficient sensor Network protocol (TEEN). This protocol combines the hierarchical technique with a data-centric approach. The main goal is to cope with sudden changes in the sensed attributes such as temperature. It works in reactive mode so energy is efficiently preserved as data transmission is done less frequently. It uses a 2-tier clustering hierarchy as shown in figure below:

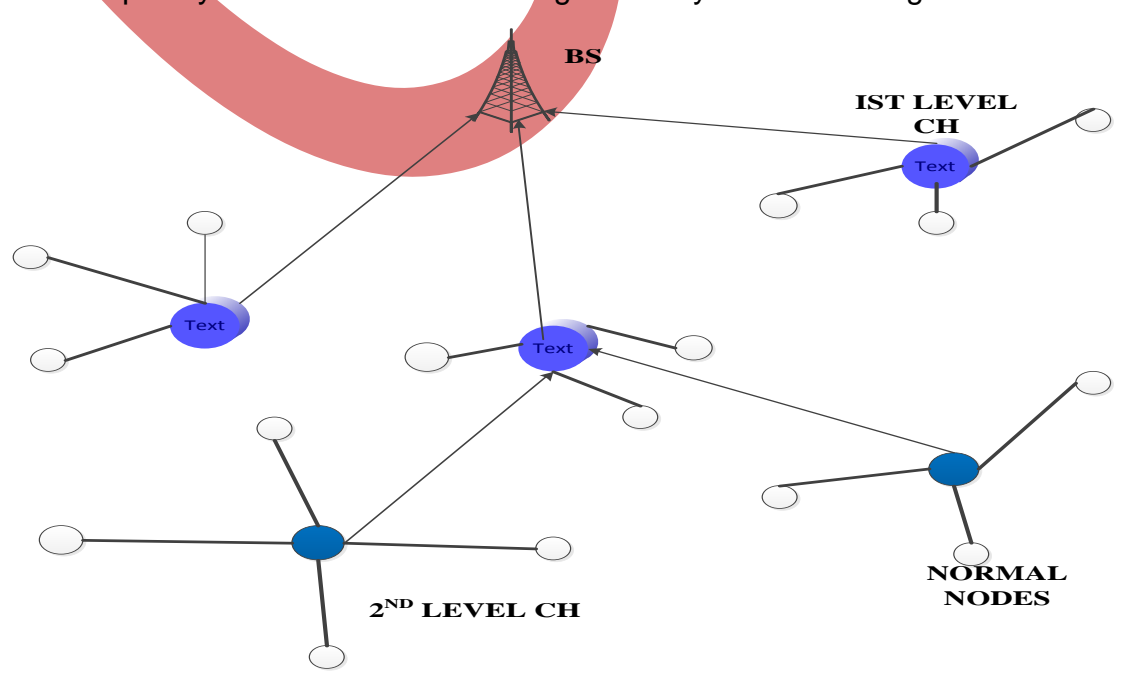

Figure 3: 2-Tier clustering Topology in TEEN

It uses two thresholds; hard threshold is value for sensed attribute. It is the absolute value of the attribute beyond which, the node sensing this value must switch on its transmitter and report to its $\mathrm{CH}$. Soft threshold is a small change in the value of the sensed attribute which triggers the node to switch on its transmitter and transmit. TEEN has the following 
advantages: (i) Based on the two thresholds, data transmission can be controlled commendably, i.e., only the sensitive data we demand can be transmitted, so that it reduces the energy transmission consumption and improves the effectiveness and usefulness of the receiving data; (ii) TEEN is complement for reacting to large changes in the sensed attributes, which is suitable for reactive scenes and time critical applications.

However, there exist a few drawbacks in TEEN as follows: (i) It has limited implementation area ,may not be applicable to applications like periodic reporting since data may not be available at all if the values of the attributes may not reach the threshold. (ii) The data may lose if $\mathrm{CHs}$ are not in the communication range of each other, because information propagation is accomplished only by $\mathrm{CHs}$.

In 2007, Amir Sepasi Zamati et. al. proposed [13] An Energy Efficient Protocol with Static Clustering for Wireless Sensor Networks (EEPSC).It is also based on the LEACH and comprise of three phases : (i) Setup Phase, (ii) Responsible Node Selection Phase and (iii) Steady State Phase. The main difference between LEACH and EEPSC is dynamic clustering and taking temporary $\mathrm{CHs}$ in Responsible Node Selection phase which are going to help in choosing the best $\mathrm{CH}$ within a cluster which will increase the network lifetime. It is hierarchical based static routing protocol in which cluster formation is initially predetermined by Base Station (BS) thus removing the complexity overhead due to dynamic clustering. In Setup phase, it is assumed that BS knows the locations of all the sensor nodes .In this scheme the BS sends $\mathrm{k}-1$ messages (where $\mathrm{k}$ is the desired number of clusters set initially) with different transmission powers the sensor nodes who listens to the messages will respond by sending the Join-Request message. For instance if BS broadcasts $k=1$ message and the nodes which hear this message set their cluster ID to $k=1$ and similarly for $k-1$ messages. The sensor nodes which are not joined to any cluster will set $k$ as their cluster ID and will inform to BS.

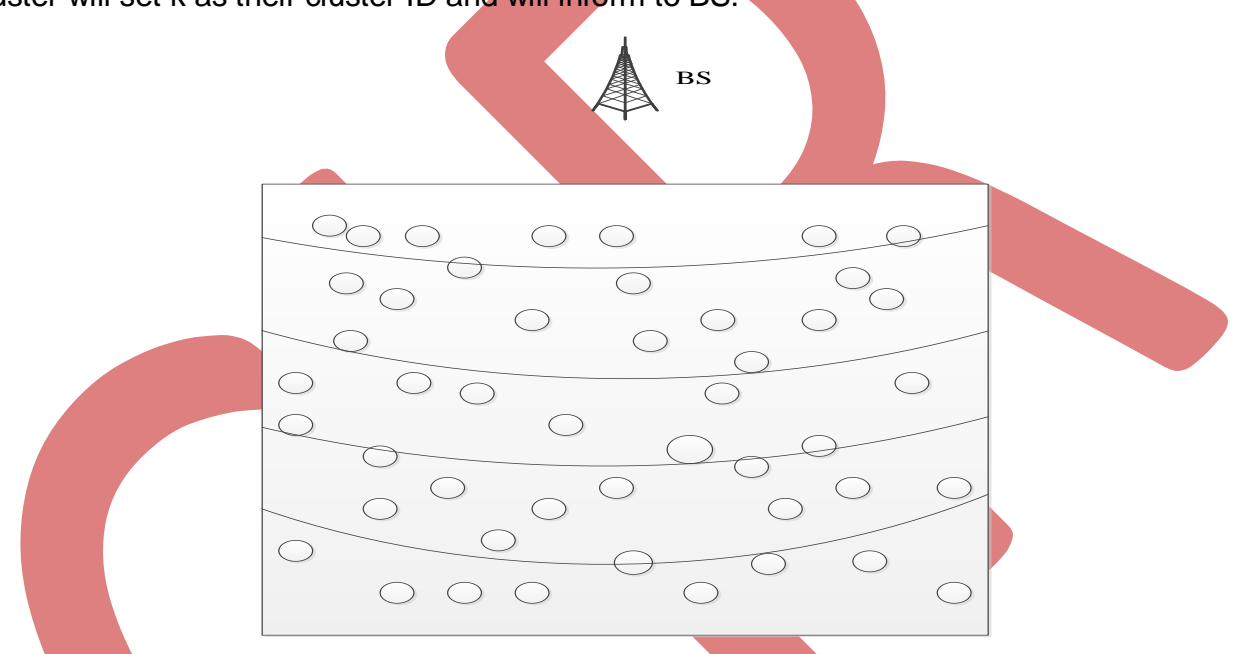

Figure 2: Network area is divided into 4 clusters with 3 messages sent by BS.

It uses CSMA (Carrier Sense Multiple Access ) for sending Join-Request messages to BS in order to reduce collision so that energy can be preserved which will enhance the network lifetime in return. BS selects one Temporary Cluster Head $(\mathrm{T}-\mathrm{CH})$ randomly for each cluster and forwards it to clusters in whole network. It also sends TDMA (Time Division Multiple Access) based schedule for all nodes in each cluster. Sensor nodes will send the data only in their allotted time slot in TDMA. In next phase, the round for selection of $\mathrm{CH}$ begins all the nodes will send energy levels to the $\mathrm{T}-\mathrm{CH}$, it then compares residual energy level of all the nodes and the node with highest energy level is selected as the $\mathrm{CH}$ for that cluster during current round. And the node with lowest energy level is selected as T-CH for next round. And in Steady Phase the nodes will send data to $\mathrm{CH}$ in their allotted time slot. The $\mathrm{CH}$ will aggregate data and perform some computation such that only the important data must be transmitted to BS directly without any hop count in between.

The main limitation of EEPSC the nodes located at the boundary of the cluster will consume more energy whenever node with utmost energy is selected as $\mathrm{CH}$ but resides on the boundary opposite to the node in that cluster. It will lead to intracommunication overhead, thus energy is more consumed will lead to early dead nodes.

In 2011 S. K. Chaurasiya et. al.[14] came out with An Enhanced Energy Efficient Protocol with Static Clustering (EEEPSC) based on existing scheme EEPSC by modifying it in order to remove the limitation of EEPSC. Cluster head is chosen not only on the basis of highest residual energy as well as the relative location of the node in cluster. The main motive of this scheme is to select the high residual node which is approximately central in the cluster. With the result intracluster communication is overhead is minimized. The phases are similar to EEPSC with some modifications like BS computes the mean position of node distribution $\left(\right.$ Pmean $\left._{i}\right)$ of every cluster where $i$ is the Cluster ID. And distance from mean position to every node in every corresponding cluster very computed by BS (dmean $\left.{ }_{i}^{j}\right)$.And node with highest residual energy and smallest mean distance will be selected as $\mathrm{CH}$ based on (Eresidual/dmean). The node with second highest residual energy is selected as $\mathrm{T}-\mathrm{CH}$ for next round in each cluster.

This energy efficient protocol has shown better simulation results as compared to LEACH and EEPSC.As per the results compared, the message received in EEEPSC is more than EEPSC. At the end of network lifetime the messages received at BS through EEEPSC is 17478 while as in EEPSC it is about 16157.And regarding the last node death, in EEPSC last 
node dies after 800 seconds while in EEEPSC it improves the lifetime by about 25\% i.e. node dies after 1000 seconds. As the future extraction, EEEPSC can be extended to heterogeneous event driven applications in wireless sensor networks.

\section{PROPOSED SCHEME}

An extension to existing efficient scheme (EEPSC) is studied in this proposal. As EEPSC assumes all the nodes in the network of same energy. We will examine the usage of heterogeneous sensor nodes especially clusters in the clusters for away from the Base Station. If we consider the total number of sensors in deployed area to be $\mathrm{n}$ and the $\mathrm{m}$ be the fraction of nodes who are having a times more energy than normal nodes $\left((1-m){ }^{*} n\right)$. These advanced nodes are assumed to be uniformly distributed in the region not nearer to BS.

As in EEEPSC, BS is located far away from the deployed region so the $\mathrm{CHs}$ in farther clusters has to transfer data directly to Base Station will take more energy than $\mathrm{CH}$ s nearer to BS. Our aim is to place the BS at an appropriate position in the deployed field such energy balance will be maintained in all clusters and clusters away from BS will take advantage of advanced node placed in those clusters such that network lifetime can be further enhanced.

\section{COMPARISON TABLE}

The routing schemes can be easily compared based on various parameters like single or multi hop network techniques, type of routing static or dynamic and mobility. The Table 1 gives brief comparison between various energy efficient routing protocols that can be used in wireless sensor networks.

\begin{tabular}{|c|c|c|c|c|c|}
\hline $\begin{array}{l}\text { TYPE OF } \\
\text { ROUTING }\end{array}$ & $\begin{array}{c}\text { SINGLE/MUL } \\
\text { TIHOP }\end{array}$ & & & $\begin{array}{l}\text { STATIC/DYNAM } \\
\text { IC ROUTING }\end{array}$ & $\begin{array}{l}\text { NETWORK } \\
\text { LIFETIME (last } \\
\text { Node dies in } \\
\text { Seconds) }\end{array}$ \\
\hline LEACH & Singl & & & Dynamic & 650 \\
\hline EAR & & CSMA & Lo & Dynamic & NA \\
\hline LEACH-C & Single & TDMA & & Static & NA \\
\hline PEGASIS & Multi- & Any MAC protocol & & Chaining & 1096 \\
\hline $\begin{array}{l}\text { OPTIMAL } \\
\text { WSN }\end{array}$ & & Any MAC protocol & & Dynamic & 801.82 \\
\hline HEED & Multi-Hop & NA & Moderate & Dynamic & NA \\
\hline EECS & Single Hop & CSMA & Moderate & Dynamic & 790 \\
\hline TEEN & Multi-Hop & NA & High & Dynamic & NA \\
\hline EEPSC & Single Hop & CSMA/TDMA & Moderate & Static & 800 \\
\hline EEEPSC & Single Hop & CSMA/TDMA & High & Static & 1000 \\
\hline
\end{tabular}

Table 1: Comparison of Various Energy Efficient Hierarchical (Clustering) Protocols

\section{REFERENCES}

[1] Akyildiz, I.F.; Su, W.; Sankarasubramaniam, Y.; Cayirci, Wireless sensor networks: A survey. Computer Network. 2002, 38, 393-422. 
[2] F.L Lewis "Wireless Sensor Network", Associate Director for research Head, Advanced Controls,Sensors, and MEMS Group, Automation and Robotics Research Institute, The University of Texis at Arlington Email Lewis@uts.edu.

[3] Al-Karaki , J.N.; and Kamal, A.E.; "Routing Techniques in Wireless Sensor Networks: A Survey", IEEE Wireless Communication Vol.11, No.6, Dec.2004, pp. 6-28.

[4] Xuxun Liu," A Survey on Clustering Routing Protocols in Wireless Sensor Networks ",Sensors 2012, 12, 1111311153; doi:10.3390/s120811113.

[5] Heinzelman, W.R.; Chandrakasan, A.;and Balkrishnan, H. ;"An Application-Specific Protocol Architecture for Wireless Micro sensor Networks", IEEE Trans. Wireless Communication, Vol. 1, No. 4, Oct. 2002, pp. 660-670.

[6] M. Younis, M. Youssef and K. Arisha, "Energy-Aware Routing in Cluster-Based Sensor Networks", Proceedings of the 10th IEEE/ACM International Symposium on Modeling, Telecommunication Systems (MASCOTS2002), Fort Worth, TX, pp.129-136, January 2002.

[7] Abad Mortaza, Mohammad Ali ,"Modify LEACH Algorithm for WSN", IJCSI-2011.

[8] Lindsey, S.; Raghavendra, C.; Sivalingam, K.M. Data gathering algorithms in sensor networks using energy metrics (PEGASIS), IEEE Trans. Parallel Distrib. Syst. 2002, 13, 924-935.

[9] Prommak Chutima ,Moshirun Sujitra,"Optimal WSN Design for Efficient Energy Utilization”, Workshop of ICAINA published in IEEE computer society 2011,814-819

[10] Younis, O.; Fahmy, S. HEED: A Hybrid Energy-Efficient, distributed clustering approach for ad-hoc sensor networks. IEEE Transaction. Mobile Comput. 2004, 3, 366-379.

[11] Ye, M.; Li, C.; Chen, G.; Wu, J. EECS: An Energy Efficient Clustering Scheme in Wireless Sensor Networks. In Proceedings of the 24th IEEE International Performance, Computing, and Communications Conference (IPCCC), Phoenix, AZ, USA, 7-9 April 2005; pp. 535-540.

[12] Manjeshwar, E.; Agrawal, D.P. TEEN: A Routing Protocol for Enhanced Efficiency in Wireless Sensor Networks. In Proceedings of the 15th International Parallel and Distributed Processing Symposium (IPDRS), San Francisco, CA, USA, 23-27 April 2001; pp. 2009-2015.

[13] Zahmati, A.S.; Abolhassani, B.;.Shirazi, A. A.B; and Bahitiari, A.S; "An Energy-Efficient Protocol with Static Clustering for Wireless Sensor Networks", International Journal of Electronics, Circuit, and Systems Vol. 1, No. 2, May. 2007, pp. 135-138.

[14] Chaurasiya S.K,Pal T., Bit S.D,"An Enhance Energy Efficient Protocol with Static Clustering for WSN" , ICOIN 2011, IEEE-2011,pp 58-63 